\title{
Coledocolitiasis primaria en situs inversus total
}

\author{
Primary choledocolithiasis in situs inversus totalis \\ Sergio Morales-Polanco, ${ }^{*}$ Oscar I Ortiz-Ruvalcaba, ${ }^{*}$ Juan de Dios Díaz-Rosales*,‡
}

\section{Palabras clave: Coledocolitiasis, situs inversus, colangiopancreatografía retrógrada endoscópica, endoscopia. \\ Keywords: Choledocholithiasis, situs inversus, cholangiopancreatography, endoscopic retrograde, endoscopy.}

\footnotetext{
* Servicio de Endoscopia Gastrointestinal/Hospital General de Zona No. 35, Instituto Mexicano del Seguro Social.

₹ Departamento de Ciencias Médicas/ Universidad Autónoma de Ciudad Juárez.
}

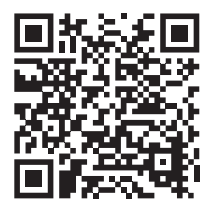

\section{RESUMEN}

El situs inversus totalis es una rara entidad congénita caracterizada por la transposición derecha-izquierda de las vísceras de tórax y abdomen. Se presenta el caso de paciente femenino de 58 años con antecedente de colecistectomía hace 18 años (donde se realizó diagnóstico de situs inversus), se presentó a urgencias con un cuadro de ictericia obstructiva. Con el antecedente quirúrgico y el conocimiento previo de su condición, se realizó un abordaje por imagen y un tratamiento endoscópico satisfactorio. La colelitiasis y el situs inversus son una rara combinación de entidades, este binomio nos recuerda que en medicina no existen conceptos absolutos.

\section{ABSTRACT}

Situs inversus totalis is an inherent condition characterized by the transposition of thoracic and abdominal viscera. We present a clinical case of a 58 years-old female, with medical history of cholecystectomy 18 years ago (and situs inversus), that currently presents with obstructive jaundice. With her surgical history and the prior knowledge of her condition, we perform an endoscopic treatment with satisfactory results. Cholelithiasis and situs inversus are a rare combination of conditions, this binomial remember us that in medicine pathognomonic symptoms, does not exist.

\section{INTRODUCCIÓN}

$\mathrm{E}^{\prime}$ situs inversus totalis se considera una entidad congénita no patológica caracterizada por la transposición derecha-izquierda de la totalidad de las vísceras del tórax y abdomen, ${ }^{1}$ tiene una frecuencia de 1:5,000-10,000 nacidos vivos. ${ }^{2}$ Esta rara condición suele poner en dilemas clínicos a los médicos ante una urgencia habitual.

La colelitiasis es un problema de salud pública y se podría considerar como parte de un problema metabólico y degenerativo. ${ }^{3}$ La coledocolitiasis es una complicación frecuente de la colelitiasis y genera más costos y otras posibles complicaciones en su tratamiento.

Presentamos el caso de una paciente con situs inversus y coledocolitiasis primaria que fue tratada con éxito mediante una colangiografía retrógrada endoscópica (CPRE).

\section{PRESENTACIÓN DEL CASO}

Paciente femenino de 58 años que fue ingresada a urgencias por ictericia y dolor en cuadrante superior izquierdo de tres días de evolución. Había cursado con el dolor tipo cólico desde hace tres meses que empeoraba después de consumir alimentos (grasos) y que mejoraba con la toma de antiespasmódicos. A la exploración física presentó una temperatura de $37.9^{\circ} \mathrm{C}$, frecuencia respiratoria 18 por minuto, frecuencia cardiaca 88 por minuto y tensión arterial 145/97 mmHg. Laboratorios a su ingreso: leucocitosis $13 \times 10^{9}$, neutrofilia de $78.3 \%$, bilirrubina total (BT) $10.3 \mathrm{mg} / \mathrm{dL}$, bilirrubina directa (BD) $7.3 \mathrm{mg} / \mathrm{dL}$, bilirrubina indirecta (BI) $2.7 \mathrm{mg} / \mathrm{dL}$, aspartato aminotransferasa (AST) $189 \mathrm{UI} / \mathrm{L}$, alanino aminotransferasa (ALT) 98 $\mathrm{UI} / \mathrm{L}$, fosfatasa alcalina (FA) $350 \mathrm{UI} / \mathrm{L}$, gamma glutamiltranspeptidasa (GGT) $630 \mathrm{UI} / \mathrm{L}$, amilasa en $148 \mathrm{UI} / \mathrm{L}$ y lipasa de $47 \mathrm{UI} / \mathrm{L}$.

La paciente tenía antecedente de laparotomía por abdomen agudo (hace 18 años) con el hallazgo de situs inversus y colecistitis, por lo que le realizaron una colecistectomía sin aparentes complicaciones. Con el antecedente quirúrgico, los hallazgos descritos por la paciente y el tiempo de evolución se

Citar como: Morales-Polanco S, Ortiz-Ruvalcaba OI, Díaz-Rosales JD. Coledocolitiasis primaria en situs inversus total. Cir Gen. 2021; 43 (1): 5-8. https://dx.doi.org/10.35366/103907 
diagnosticó ictericia obstructiva y se sospechó de una coledocolitiasis primaria (por el tiempo de evolución desde el procedimiento previo a su padecimiento actual), por lo que se solicitaron estudios de imagen. La tomografía de abdomen confirmó el diagnóstico de situs inversus, dilatación de la vía biliar y lito único de $15 \mathrm{~mm}$ en el colédoco (Figura 1).

La paciente fue sometida a una CPRE, bajo anestesia general, con posición en decúbito prono y con el endoscopista al lado derecho de la mesa. Se introdujo el duodenoscopio hasta el estómago, se realizó una rotación de $180^{\circ}$ contra las manecillas del reloj y se introdujo en el píloro. Con el duodenoscopio en la segunda porción del duodeno y en asa larga (debido a la dificultad para el posicionamiento correcto) se realizó una visualización cuidadosa y se encontró una papila nativa que se canuló con dirección a la una (con respecto a las manecillas del reloj) hacia la vía biliar (en este caso, esta dirección "inversa" nos otorga una correcta canulación). Se realizó una colangiografía que mostró la dilatación del colédoco y defectos de llenado, por lo que se realizó una esfinterotomía (hasta el pliegue transverso) y dilatación del esfínter de Oddi. Se realizaron tres barridos con catéter de balón de extracción, obteniendo abundante detritus biliar (Figura 2) y un correcto vaciamiento del medio de contraste al finalizar la CPRE. La paciente fue egresada el mismo día del procedimiento y durante las evaluaciones posteriores (durante un año) se encuentra asintomática y con estos laboratorios a su último seguimiento: BT 1.2 $\mathrm{mg} / \mathrm{dL}$, BD $0.6 \mathrm{mg} / \mathrm{dL}, \mathrm{BI} 0.6 \mathrm{mg} / \mathrm{dL}$, AST 46 UI/L, ALT 56 UI/L. No se encontró evidencia de otra causa orgánica de la ictericia, por lo que se da alta médica.

\section{DISCUSIÓN}

El situs inversus es una rara condición que implica dificultades diagnósticas y terapéuticas en enfermedades comunes. Aunque esta condición no es un factor de riesgo de colelitiasis, sí incrementa la dificultad y los riesgos durante los procedimientos invasivos como la CPRE debido a que la anatomía se debe considerar en espejo. ${ }^{4}$

No hay consenso de como realiar la CPRE, ni tampoco hay una tecnica estanadr de oro para trstar la coledocolitiasis en situs inversus. La CPRE la realizara en endoscopista o cirujano como se sienta más seguro o practicamente como se lo permitan las condiciones en ese momento.

Aunque es una entidad poco frecuente, el paciente adulto puede tener conocimiento de su condición y no darle la importancia requerida (incluso olvidarlo) hasta que es redescubierto durante el abordaje diagnóstico por alguna
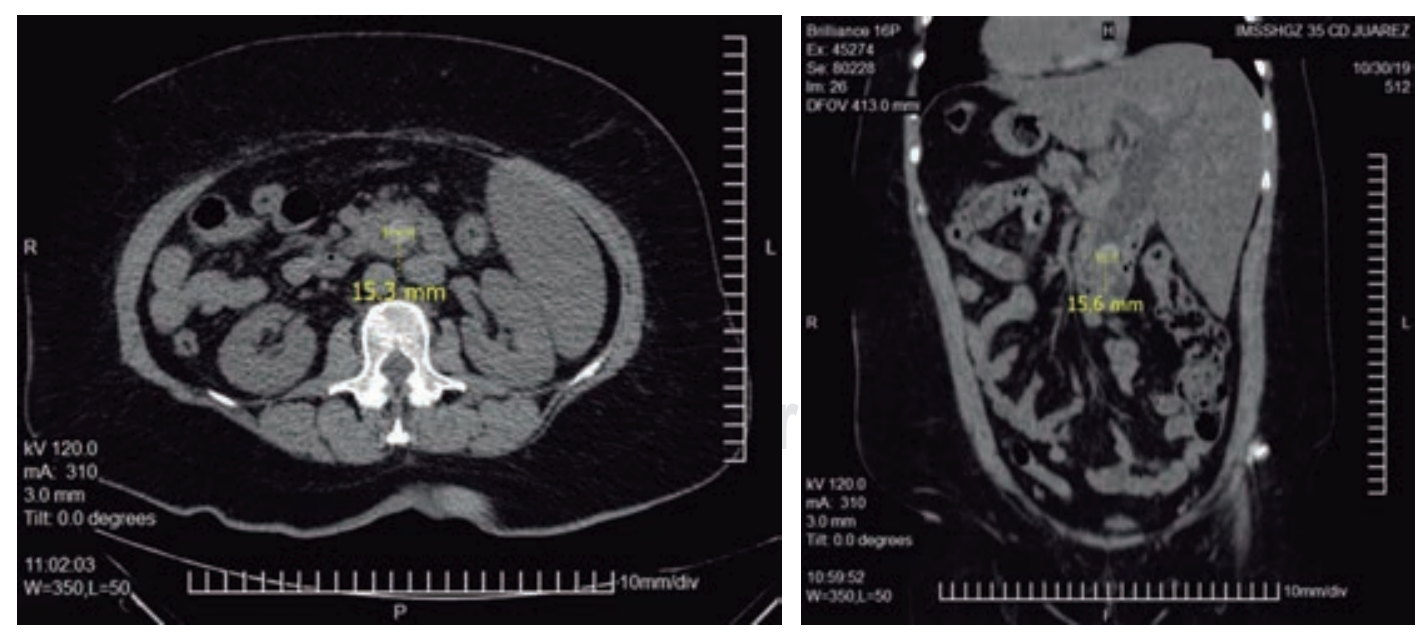

Figura 1: Tomografía computarizada en cortes axial y coronal que confirma el diagnóstico de situs inversus totalis y donde se observa dilatación de la vía biliar y litiasis de $15 \mathrm{~mm}$. 

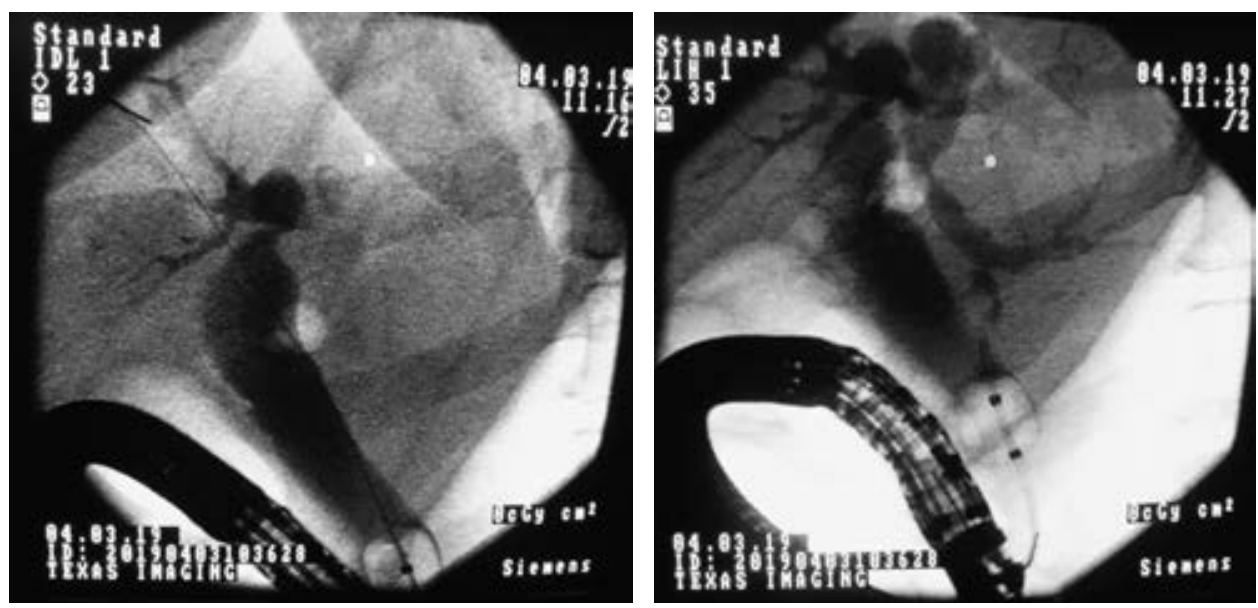

Figura 2: Colangiografía donde se observa la dilatación de la vía biliar y el barrido con sonda de balón.

enfermedad común (apendicitis, colecistitis, diverticulitis, etcétera). ${ }^{5}$

El paciente ictérico con situs inversus deberá ser sometido a un abordaje diagnóstico y terapéutico prácticamente igual al de un paciente con anatomía normal. Los estudios de imagen como el ultrasonido de abdomen y la tomografía computarizada harán el diagnóstico en la mayoría de los casos. Sin embargo, sí se requiere del conocimiento previo de la anatomía biliar, la colangiorresonancia magnética (CRM) nos dará información detallada para planear el abordaje terapéutico más conveniente. ${ }^{6}$

Aunque no existe un consenso acerca de su abordaje terapéutico, la CPRE es el tratamiento inicial de la mayoría de los autores y, por lo tanto, podríamos considerarla como el de elección. Aunque algunos autores consideran que la posición (del endoscopista) a la derecha de la mesa, no es necesaria para la realización de la CPRE en situs inversus, ${ }^{7}$ la realidad es que no existe un consenso y lo recomendable es hacer el procedimiento como el endoscopista o cirujano se sienta más seguro en su técnica o las condiciones en ese momento se lo permitan. Lo anterior, prácticamente justifica todas las maniobras realizadas para alcanzar el objetivo (que es extraer el lito o "limpiar" la vía biliar) con la mayor seguridad. Debemos enfatizar que, debido a la dificultad técnica, un procedimiento endoscópico no-satisfactorio no deberá ser considerado una falla ${ }^{8}$ y en ese caso se podrá abordar al paciente de manera quirúrgica. ${ }^{9-11}$

La colelitiasis y el situs inversus son una rara combinación de entidades, este binomio nos recuerda que en medicina no existen conceptos absolutos.

\section{REFERENCIAS}

1. Hu Y, Zeng H, Pan XL, Lv NH, Liu ZJ, Hu Y. Therapeutic endoscopic retrograde cholangiopancreatography in a patient with situs inversus viscerum. World J Gastroenterol. 2015; 21: 5744-5748.

2. Fiocca F, Donatelli G, Ceci V, Cereatti F, Romagnoli F, Simonelli $\mathrm{L}$ et al. ERCP in total situs viscerum inversus. Case Rep Gastroenterol. 2008; 2: 116-120.

3. Díaz-Rosales JD, Alcocer-Moreno JA, Enríquez-Domínguez L. Síndrome metabólico y colecistitis complicada en mujeres adultas. Arch Med. 2016; 16: 304-311.

4. Hu L, Chai Y, Yang X, Wu Z, Sun H, Wang Z. Duodenoscope combined with laparoscopy in treatment of biliary stones for a patient with situs inversus totalis: a case report. Medicine (Baltimore). 2019; 98: e14272.

5. Rosen H, Petrosyan M, Mason RJ. Cholecystitis in situs inversus totalis. Radiol Case Rep. 2008; 3 (4): 226.

6. Eddes EH, Koster K. MRCP in situs inversus. Dig Surg. 2002; 19: 2.

7. Sheikh I, Heard R, Tombazzi C. Technical factors related to endoscopic retrograde cholangiopancreatography in patients with situs inversus. Gastroenterol Hepatol (NY). 2014; 10: 277-278.

8. Morales-Rodríguez JF, Corina Cotillo E, Moreno-Loaiza O. Surgical treatment of choledocholithiasis in a patient with situs inversus totalis: a case report and literature review. Medwave. 2017; 17 (06): e7002.

9. Alzahrani HA, Yamani NM. Gallbladder agenesis with a primary choledochal stone in a patient with situs 
inversus totalis. Am J Case Rep. 2014; 15: 185-188.

10. Takalkar YP, Koranne MS, Vashisst KS, Khedekar PG, Garale MN, et al. Laparoscopic cholecystectomy with choledochoduodenostomy in a patient with situs inversus totalis. J Minim Access Surg. 2018; 14: 241243.

11. Moyon MA, Rojas CL, Moyon FX, Aguayo WG, Molina GA, et al. Acute cholecystitis and residual choledocholithiasis in situs inversus patient, successful approach and ERCP a case report from Ecuador. Ann Med Surg (Lond). 2020; 54: 101-105.

Consideraciones y resposabilidad ética: Privacidad de los datos. De acuerdo a los protocolos establecidos en el centro de trabajo de los autores, éstos declaran que han seguido los protocolos sobre la privacidad de datos de pacientes y preservado su anonimato. El consentimiento informado del paciente referido en el artículo se encuentra en poder del autor principal.

Financiamiento: No se recibió apoyo financiero para la realización de este trabajo.

Conflicto de intereses: Los autores declaran que no existe ningún conflicto de intereses en la realización del trabajo.

\section{Correspondencia:}

Sergio Morales-Polanco

E-mail: sergiomp90@hotmail.com 